\title{
Arylsulfonyl Chloride Induced Setting of Dolphinfish Flesh Sol
}

\author{
Eiji Niwa*, Teruo NaKayama*, and Iwao HamadA* \\ (Received August 6, 1980)
}

\begin{abstract}
In order to investigate the participation of the hydrophobic groups into muscle protein on its gelation on heating at low temperature, viz. Setting, the elasticity was measured on the flesh sol from dolphinfish, Coryphaena hippurus, containing p-toluenesulfonyl chloride (TSCl) or $\alpha$-naphthalenesulfonyl chloride (NSCl). Washed mince was ground for $25 \mathrm{~min}$ with $\mathrm{TSCl}$ or $\mathrm{NSCl}(0.3 \%$ of the mince) together with $\mathrm{NaCl}(3 \%)$ and $\mathrm{NaHCO}_{3}(0.15 \%)$. With the addition of $\mathrm{TSCl}$ or $\mathrm{NSCl}$, a slight increase in the breaking stress and the decrease in $\mathrm{pH}$ by about 0.2 was observed in the flesh sol. On heating at $40^{\circ} \mathrm{C}$ or by standing at $4^{\circ} \mathrm{C}$, the flesh sol changed to gel and a remarkable increase in the breaking stress was observed, but sucrose $(10 \%)$ diminished the effect of $\mathrm{TSCl}$ or $\mathrm{NSCl}$. In the flesh sol without $\mathrm{TSCl}$ or $\mathrm{NSCl}$, however, the gelation was delayed even at $40^{\circ} \mathrm{C}$ and the breaking stress of the resulting gel was low.

The preheating of the fiesh sol containing $\mathrm{TSCl}$ or $\mathrm{NSCl}$ at $40^{\circ} \mathrm{C}$ caused to increase the breaking stress of the fish gel obtained by further heating the sol at $90^{\circ} \mathrm{C}$ for $25 \mathrm{~min}$.

From the above results, the dolphinfish flesh sol, which is classified into a hard-to-set fish, seems to come to behave like the flesh sol from the easy-to-set fish, on the addition of TSCl or NSCl.
\end{abstract}

The ease of fish flesh sol for the gelation at low temperature, Setting, is species-specific, as well known, and dolphinfish, Coryphaena hippurus, is classified into a group hardest to set. ${ }^{\mathrm{i}}$ Most recently, pig and hen muscle proteins into which the aryl groups were introduced, have been found to set to gel upon heating at $40^{\circ} \mathrm{C}^{2)}$ despite of the hard-to-set characteristics. ${ }^{1,33}$

This paper deals with that the dolphinfish flesh sol comes to behave like the flesh sol from the easy-to set fish on the addition of the arylsulfonylating reagent.

\section{Experimental}

\section{Gel Preparation}

Dolphinfish was obtained at market, which had been fished at the coast of Daiohzaki the day before and brought under chilling. The white dorsal and lateral muscles were collected, minced, and immersed in 7 volumes of ice-water. The sewage was removed by centrifugal filter. After washing 3 times, the mince was ground with ice-water $(20 \%$ of the mince), $\mathrm{NaCl}(3 \%)$ and $\mathrm{NaHCO}_{8}(0.15 \%)$ for $5 \mathrm{~min}$ in a mechanical mortar, and ground further for $20 \mathrm{~min}$ with or without $p$-toluenesulfonyl chloride (TSCl) or $\alpha$-naphthalenesulfonyl chloride (NSCl), of which the amounts were summarized in Table 1. In the case when sucrose
Table 1. Composition of dolphinfish flesh sol*

\begin{tabular}{lccc}
\hline \multirow{2}{*}{ Sol } & \multicolumn{3}{c}{ Additives $(\%$ of the mince) } \\
\cline { 2 - 4 } & TSCl & NSCl & Sucrose \\
\hline C & - & - & - \\
CS & - & - & 10 \\
T-1 & 0.1 & - & - \\
T-3 & 0.3 & - & - \\
TS-3 & - & - & 10 \\
N-3 & - & 0.3 & - \\
NS-3 & - & 0.3 & 10 \\
\hline Moreover, water $(20 \%$ of the mince $), \mathrm{NaCl}(3.0 \%)$ \\
and NaHCO $(0.15 \%)$ were added.
\end{tabular}

was added, the mince was ground with it further for $10 \mathrm{~min}$. The resulting flesh sol was packed into polyvinylidene chloride tube and left to gel in an waterbath at definite temperatures.

\section{Elasticity Measurement}

After cooled with running water, the gel was cut to slices together with the tube (Thickness: $2.5 \mathrm{~cm}$, Diameter: $3 \mathrm{~cm}$ ). Each slice was subjected to the breaking test with the spherical plunger (Diameter: $6 \mathrm{~mm}$, Speed: $14 \mathrm{~mm} / \mathrm{min}^{4}$, after removing the surrounding tube-ring. In the case of the viscous sample, the test was carried out without removing the tube-ring, and the breaking stress was conveniently obtained from the stress at the inflection point of stress-strain curve. $\mathrm{pH}$ of the sample was

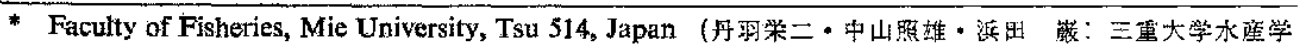
部). 
determined by thrusting an electrode into it (TOA Electronics GST-115C).

\section{Results}

Fig. 1 shows the changes in the stress-strain curve for two types of dolphinfish sol during heating at $40^{\circ} \mathrm{C}$, one contains TSCl (T-3) and another does not (C). It is obvious that both flesh sols set to gel upon heating, but the former's elastic modulus increases more rapidly than the latter's. Fig. 2 shows the changes in the breaking stress and $\mathrm{pH}$ of the flesh sol during heating at $40^{\circ} \mathrm{C}$. The increase in the breaking stress is remarkable for the flesh sol containing TSCl or NSCl (T-3 and N-3), but it is not so remarkable for that with smaller amount of the reagent (T-1). This increase is diminished by the addition of sucrose (TS-3, NS3 and $C S$ compared with $T-3, N-3$ and $C$, respectively).

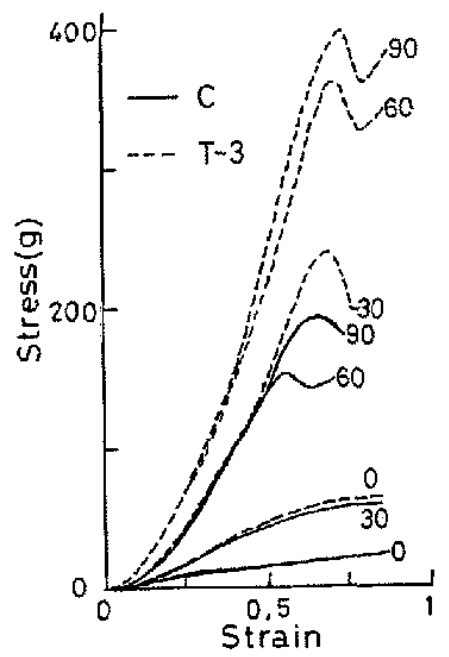

Fig. 1. Changes in stress-strain curve for dolphinfish flesh sol upon heating at $40^{\circ} \mathrm{C}$.

Symbols (C, T-3) show types of sol, of which compositions should be referred to Table 1. The figures appended to the curves show heating times (min).

Fig. 3 shows the changes in the breaking stress of the flesh sol during standing in a cold room at $4^{\circ} \mathrm{C}$. A remarkable increase in the breaking stress is observed on the flesh sol containing $\mathrm{TSCl}$ or NSCl (T-3 and N-3). As shown through Figs. $1-3$, even before heating or standing, the breaking stress is higher and pH is lower in the flesh sol containing $\mathrm{TSCl}$ or $\mathrm{NSCl}$ than in that without

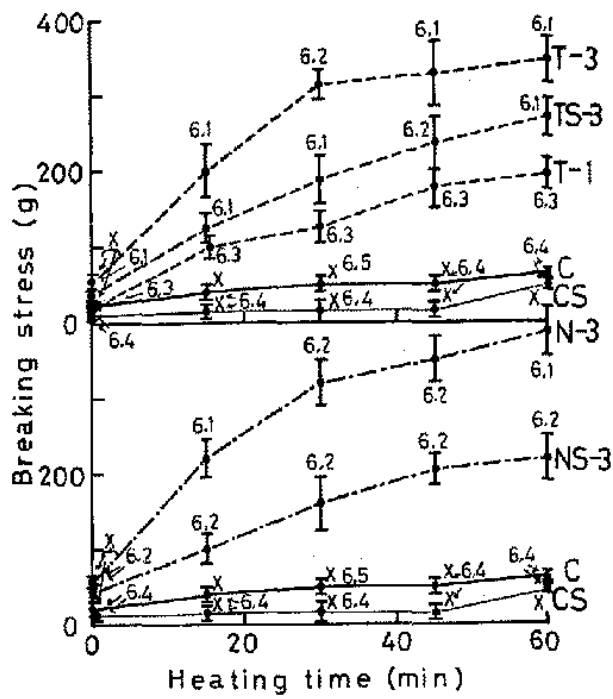

Fig. 2. Changes in breaking stress and $\mathrm{pH}$ of dolphinfish flesh sol during heating at $40^{\circ} \mathrm{C}$. Vertical bars and figures show standard deviation and $\mathrm{pH}$, respectively. Symbol, $\mathrm{x}$, implies the value obtained from the stress at the inflection point of stress-strain curve. Types of sol (C, T-1, N-3 etc.) should be referred to Table 1 .

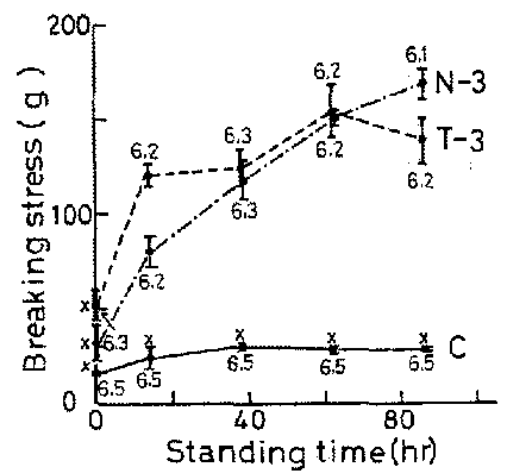

Fig. 3. Changes in breaking stress and $\mathrm{pH}$ of dolphinfish flesh sol during standing at $4^{\circ} \mathrm{C}$. Vertical bars, figures and symbol, $x$, are interpreted in Fig. 2. Type of sol (C, T-3 etc.) should be referred to Table 1.

them. During heating at $40^{\circ} \mathrm{C}$, pH of each flesh sol remains unchanged, but it falls gradually in one of the former sol (N-3) with the passage of standing at $4^{\circ} \mathrm{C}$.

Fig. 4 shows the effect of the preheating of the flesh sol at $40^{\circ} \mathrm{C}$ on the breaking stress and $\mathrm{pH}$ of the fish gel obtained by further heating at $90^{\circ} \mathrm{C}$ for $25 \mathrm{~min}$. In the fish gel containing $\mathrm{TSCl}$ or $\mathrm{NSCl}$ 


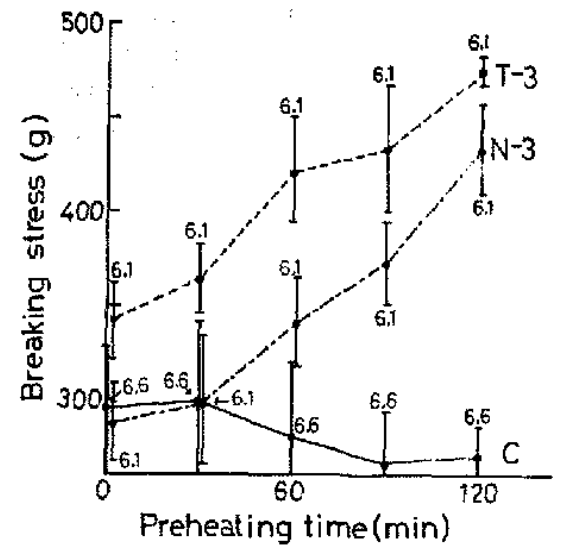

Fig. 4. Effect of preheating of dolphinfish flesh sol at $40^{\circ} \mathrm{C}$ on breaking stress and $\mathrm{pH}$ of fish gel obtained by further heating at $90^{\circ} \mathrm{C}$ for $25 \mathrm{~min}$. Vertical bars and figures are interpreted in Fig. 2. Type of gel (C, N-3 etc.) should be referred to Table 1.

( $\mathrm{T}-3$ and $\mathrm{N}-3$ ), the breaking stress increases in proportion to the preheating periods, while in the fish gel without them $(\mathrm{C})$, the effect of preheating is not observed. The $\mathrm{pH}$ of the gel drops on the addition of $\mathrm{TSCl}$ or $\mathrm{NSCl}$.

\section{Discussion}

Hen actomyosin reacted with $\mathrm{TSCl}$ or $\mathrm{NSCl}$ (30\% of the protein) under the conditions of $4^{\circ} \mathrm{C}$ and $\mathrm{pH} 7.5$ to give salt-insoluble product ${ }^{3)}$. The product retained still the faculty of super-precipitation, but showed negative value in ATP-sensitivity. Such a strange phenomenon in the latter could be interpreted not as a result of the denaturation of the actomyosin, but as a result of the introduction of the extraordinarily hydrophobic groups. Therefore, in the case that the arylation was carried out with $\beta$-naphthoquinone-4,7-disulfonate leaving a strong hydrophilic sulfonyl group on the 7 position of its ring after reaction, the arylated actomyosin did not lose its salt-solubility and retained the normal biological activities. ${ }^{2)}$ In the present studies, the reaction was carried out with slight modifications, that is, the fiesh sol instead of actomyosin, lower $\mathrm{pH}$ and smaller quantity of the reagent were used compared with the case of the hen actomyosin.

However, also in this case, the reagent seems to react with the mercapto, hydroxy and amino groups of the fish flesh protein, because the reactivity of
$\mathrm{TSCl}$ or $\mathrm{NSCl}$ is thought to be similar to that of 1-dimethylaminonaphthalene -5-sulfonyl chloride (dansyl-Cl) ${ }^{\text {b) }}$ which is frequently used to investigate the stereostructure of proteins, and the reaction condition mentioned above is not so differ from that for the hen actomyosin. This presumption is also supported from that the flesh sol containing the reagent was always lower in $\mathrm{pH}$ than the sol without it because of the liberated hydrogen chloride as a by-product.

The gradual drop of the $\mathrm{pH}$ of the flesh sol ( $\mathrm{N}$ 3) during standing at $4^{\circ} \mathrm{C}$ may be interpreted by the gentle reaction with the protein. The protein in the flesh sol is not likely undergo a large conformational change during the reaction with the reagent also in this case, because the biological activity remained in the case of the hen actomyosin.

At $40^{\circ} \mathrm{C}$, the flesh sol containing the reagent set to gel more rapidly and showed higher breaking stress than that without the reagent. Sucrose inhibited the increase in the breaking stress during heating. The gelation enhanced by the reagent was observed even at $4^{\circ} \mathrm{C}$. Upon preheating the fish flesh sol containing the reagent at $40^{\circ} \mathrm{C}$, the fish gel obtained by further heating at $90^{\circ} \mathrm{C}$ was strengthened in the breaking stress. On the contrary, the effect of preheating was not observed in the sol without the reagent.

The above gelling characteristics of the flesh sol containing the reagent are similar to those of the flesh sol made from the species which are supposed to be easy to set.

Especially, the inhibition of the increase in the breaking stress by sucrose and the strengthening of the fish gel by preheating the flesh sol are the distinctive features of the setting, in addition to the gelation of the flesh sol by low temperature-heating. It is noticeable that the breaking stress of the fish gel increased in spite of the remarkable drop in its pH-presumably as a result of the complete hydrolysis of the remaining reagent, because lowering $\mathrm{pH}$ is known to cause to decrease the breaking stress. ${ }^{6)}$

Even before heating or standing, the flesh sol containing the reagent was always higher than the sol without the reagent in the breaking stress. Therefore, the effect of heating or standing on the increase in the breaking stress seerns outwardly to be reduced. A similar phenomenon can be observed in the hen and actomyosins arylated with $\beta$-naphthoquinone-4,7-disulfonate and then purified by two cycles of dissolution and precipitation. ${ }^{2)}$ The arylated actomyosins were always higher in 
the relative viscosity before heating than the unarylated ones. Two reasons are thought for such the highness in the breaking stress of the fiesh sol containing the reagent. One is the increase in the elasticity and another is that in the viscosity.

If the reason is mainly due to the increase in the elasticity, the network structure would begin to be formed between the protein molecules, as soon as the hydrophobic aromatic groups are introduced to the surface of the molecules. However, further details can not be discussed here, because the elasticity and viscosity can not be calculated separately from the result of plunger test.

In any way, it is suggested that the muscle protein of the dolphinfish flesh is converted functionally into the protein from the easy-to-set species by the arylsulfonation of the cysteine, serine and lysine residues, considering the similarity of the above thermal behaviors of the dolphinfish flesh sol containing $\mathrm{TSCl}$ or NSCl to those of the fish flesh sol from the easy-to-set species.

Therefore, the aromatic amino acid residues should be noticed for the clarification of the setting also from the fact that the absorbance at near UV region is high in the myosin heavy chain prepared from the easy-to-set species. ${ }^{2)}$
The authors wish to express their sincere gratitude to Dr. S. Nogucri, Taiyo Gyogyo Co. Ltd. for his kind reading of the manuscript. This study was supported partly by the grant of Kieikai.

\section{References}

1) Y. SHLizU: in "Shiromi no Sakana to Akami no Sakana" (White Meat Fish and Red Meat Fish, ed. by Japan. Soc. Sci. Fish.), Koseisha-koseikaku, Tokyo, 1976, pp. 106-118.

2) E. Niwa, T. Nakayama, and l. Hamada: Agr, Biol. Chem., 45, 341-344 (1981).

3) E. Niwa: in "Shokuhin no Bussei" (Viscoelasticities of Foods, Vol. 3, ed. by S. Matsumoto), Shokuhin-shizai-kenkyukai, Tokyo, 1977, pp. 19. 30.

4) E. Niwa, T. Konagaya, and M. Mryake: $J$, Agr. Chem. Soc. Jap., 46, 295-301 (1972).

5) T. IKeUCHr: in "Tanpakushitsu no Kagaku" (Chemistry of Proteins, Vol. 2, ed. by K. NarITa and T. MURAs), Tokyokagakudojin, Tokyo, 1976, pp. 142-153.

6) A. TANakA, T. Mito, and Y. INoue: $J$. Fac. Fish. Pref. Univ. Mie, 7, 271-276 (1968),

7) T. Nakayama: Japan. Soc. Food, Sci. Technol, 22, 19-23 (1975).

* The breaking stress of the viscous sol determined as above is thought to be affected by its viscosity in addition to its elasticity, because the viscosity of such a sol whose curd meter pattern does not show a distinct breaking point is calculated as follows ${ }^{7}$ :

$$
\text { Viscosity }=-\frac{\text { Stress at inflection point }}{\text { Area of plunger tip } \times \text { Constant strain rate }}
$$

